\title{
Comunicação
}

[Communication]

\section{Osteossíntese de tibiotarso com miniplaca de titânio em Arara Canindé (Ara ararauna)}

\author{
[Tibiotarsus osteosynthesis' in blue-yellow-macaw (Ara ararauna) using titanium miniplate]
}

I.S. Dal-Bó ${ }^{1}$, M.M. Alievi ${ }^{1}$, L.M. Silva ${ }^{1}$, A.S. Gouvêa ${ }^{1}$, M.S. Mucillo ${ }^{1}$, E.O. Santos $^{2}$, C.A.C. Beck ${ }^{1}$

\author{
${ }^{1}$ Faculdade de Veterinária - UFRGS \\ Av. Bento Gonçalves, 9090 \\ CEP 91540-000 - Porto Alegre, RS \\ ${ }^{2}$ Médico Veterinário do Parque Pampas Safari - Gravataí, RS
}

\begin{abstract}
Os ossos longos das aves, quando comparados aos dos mamíferos, apresentam canal medular com diâmetro proporcionalmente maior e cortical fina e quebradiça devido à alta densidade de cálcio presente (Alievi et al., 2008). Helmer e Redig (2006) e Ferraz et al. (2008) afirmaram que a cortical fina e frágil das aves contribui para a menor fixação dos implantes metálicos ortopédicos. Além disso, as aves apresentam trabéculas ósseas muito finas e esparsas ao longo do comprimento ósseo e falta de osso denso na região metafisária (West et al., 1996). Segundo Helmer e Redig (2006), a menor quantidade de tecidos moles recobrindo os ossos é considerada fator limitante para a consolidação das fraturas nesses animais.
\end{abstract}

Para redução de fraturas, em ossos longos de aves, podem ser utilizados imobilização externa (Stauber et al., 2008), pinos intramedulares e suas associações (Alievi et al., 2008), fixadores esqueléticos externos (Alievi et al., 2002) e placas e parafusos (Davidson et al., 2005).

Segundo Davidson et al. (2005), as placas oferecem maior estabilidade, quando comparadas aos demais métodos de fixação, proporcionando retorno precoce à função do membro, além de serem bem toleradas pelos animais silvestres, uma vez que não há necessidade de manuseio frequente para curativos. Estes autores relataram o uso de miniplaca de 2,0mm para osteossíntese de coracoide em uma águia (Haliaeetus leucocephalus), e Guzmann et al. (2007) relataram o reparo de luxação coracoide-esternal utilizando placa em $\mathrm{T}$ de $1,5 \mathrm{~mm}$ e placa de compressão dinâmica (DCP) de 2,0mm em uma águia (Haliaeetus leucocephalus). No mesmo animal, esses autores realizaram osteossíntese de tibiotarso utilizando um pino intramedular e placa DCP de $2,7 \mathrm{~mm}$.

As miniplacas de titânio foram projetadas para serem usadas em cirurgias maxilofaciais em humanos. A utilização desses implantes, em humanos, resultou em mínima reação inflamatória e ausência de infecção periimplante, além de diminuição do desconforto pós-cirúrgico, permitindo a recuperação precoce (Sugawara et al., 2002).

Em medicina veterinária, esse tipo de implante metálico foi empregado na redução de fraturas de mandíbula e maxila de cães e gatos e, mais recentemente, em fraturas dos ossos longos em filhotes de raças toy. As placas de titânio são facilmente moldadas e, por apresentarem tamanho reduzido, tornam o procedimento menos invasivo (Bilgili e Kurum, 2003). Sua elevada elasticidade juntamente com suas propriedades anticorrosivas fizeram desse implante uma alternativa para osteossínteses em ossos longos de aves de porte médio e pequeno (Hatt et al., 2001).

O presente relato descreve a utilização de miniplaca de titânio para osteossíntese de tibiotarso em uma arara Canindé (Ara ararauna).

Recebido em 23 de março de 2011

Aceito em 6 de junho de 2011

E-mail: idb_vet@yahoo.com.br 
A ave foi atendida, em Hospital Veterinário Escola, por apresentar impotência funcional do membro pélvico esquerdo (MPE). Ao exame físico, o animal apresentava-se magro (1000g), apático e com aumento de volume firme em região diafisária de tibiotarso esquerdo. Foi sedado, utilizando-se xilazina $\left(1 \mathrm{mg} \cdot \mathrm{kg}^{-1}\right)$ e cetamina (5mg. $\mathrm{kg}^{-1}$ ) IM, e encaminhado ao Setor de Radiologia. As radiografias do tibiotarso esquerdo evidenciaram luxação da articulação femurotibial e fratura completa transversa em terço médio de diáfise com desvio de eixo ósseo e reação periosteal, indicando fratura antiga (Fig. $1-\mathrm{A})$.

$\mathrm{O}$ animal foi submentido à osteossíntese de tibiotarso esquerdo. Como medicação préanestésica, foi administrado cloridrato de metadona na dose de $0,2 \mathrm{mg} \cdot \mathrm{kg}^{-1}$, por via intramuscular. A indução anestésica foi realizada com isofluorano por meio de máscara, e o animal foi submetido à intubação orotraqueal, utilizando-se tubo endotraqueal número 3,5. A manutenção anestésica foi realizada com isofluorano e oxigênio a $100 \%$ em sistema aberto. Após a indução, a ave foi posicionada em decúbito dorsal e foi realizada a remoção manual da penas do MPE. A antissepsia foi realizada com gluconato de clorexidine a $4 \%$.

O acesso cirúrgico ao tibiotarso foi realizado por meio de uma incisão cutânea de, aproximadamente, $5 \mathrm{~cm}$, na face medial da região do tibiotarso esquerdo, seguida pela divulsão do tecido subcutâneo e musculatura. Após a identificação dos segmentos da fratura, as extremidades foram curetadas e o canal medular foi desobstruído. A fratura foi reduzida $\mathrm{e}$ estabilizada com o auxílio de uma miniplaca de $2,0 \mathrm{~mm}$ e seis miniparafusos de $2,0 \mathrm{~mm}$ de diâmetro e 9,0mm de comprimento (três no segmento proximal e três no distal) (Fig. 1-B). A musculatura foi aproximada com padrão contínuo simples e fio poliglactina 910, 4-0, a dermorrafia foi realizada com náilon monofilamentar 4-0 e com o mesmo padrão de sutura. As medicações utilizadas no pósoperatório foram cetoprofeno $2 \mathrm{mg} \cdot \mathrm{kg}^{-1}$, IM, SID, 4 dias, cloridrato de tramadol $2 \mathrm{mg} . \mathrm{kg}^{-1}$, IM, BID, 3 dias e enrofloxacina $10 \mathrm{mg} \cdot \mathrm{kg}^{-1}$, IM, SID, 10 dias. O exame radiográfico no pós-operatório imediato revelou fratura alinhada e coapatada (Fig. 1-C).

A sutura de pele foi removida em dez dias, e o animal foi mantido em repouso em gaiola individual durante quinze dias. Aos trinta dias de pós-operatório, foi realizado novo exame radiográfico, o qual evidenciou fratura consolidada e alinhada, sem presença de calo ósseo exuberante e ausência de implantes metálicos frouxos.

A luxação fêmurotibial não foi reduzida devido ao acréscimo do tempo cirúrgico, sendo postergada para uma segunda intervenção. Mesmo com a restrição dos movimentos dessa articulação, o animal manteve satisfatória função do membro, visto que o paciente conseguia apoiá-lo.

A osteossíntese com placas e parafusos tem sido relatada com sucesso em diferentes espécies de aves e em variados ossos, mas historicamente foi desencorajada devido às características das corticais ósseas (finas e quebradiças), as quais dificultariam a sua aplicação (Davidson et al., 2005). Entretanto, no caso em questão, foi verificada uma adequada fixação dos miniparafusos nas corticais do tibiotarso, sem qualquer dificuldade ou fragilização do osso. Entre as possíveis explicações para esse fato citam-se o porte do paciente, o tipo de osso (não pneumático) e as características dos parafusos utilizados. Semelhante à recomendação de Hollamby et al. (2004), optou-se pela não remoção dos implantes metálicos mesmo após a consolidação da fratura, já que o titânio é um material biocompatível e não necessita remoção, o que facilita a sua utilização em animais silvestres candidatos à reabilitação.

Segundo Davidson et al. (2005), a miniplaca, semelhante à utilizada neste relato, promoveu adequada estabilização e redução da fratura, o que permitiu um retorno precoce à função do membro, já que age contrapondo adequadamente as forças de rotação, flexão, compressão e cisalhamento que atuam no foco de fratura. Além disso, foi bem tolerada pelo paciente, visto que não necessitou de grandes cuidados pósoperatórios por ser um método interno de osteossíntese (Guzman et al., 2007). 


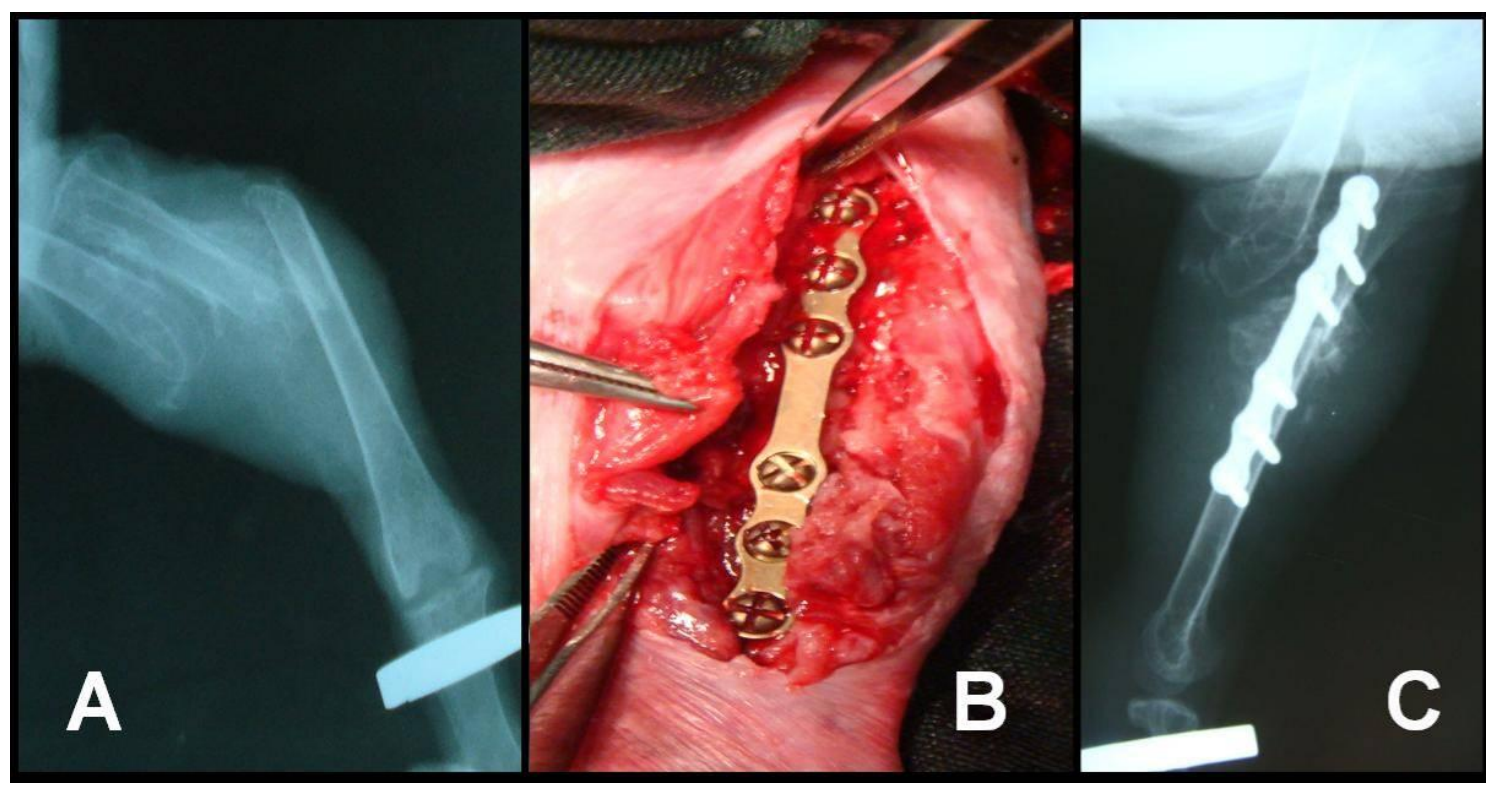

Figura 1. Osteossíntese de tibiotarso com miniplaca de titânio em Arara Canindé (Ara ararauna). A: Radiografia do tibiotarso esquerdo evidenciando luxação da articulação femurotibial e fratura completa transversa em diáfise e reação periosteal indicando fratura antiga. B: Fratura reduzida após a utilização de miniplaca de titânio de 2,0mm com seis orifícios e seis miniparafusos (três no segmento proximal e três no segmento distal). C: Radiografia do pós-operatório imediato evidenciando fratura alinhada e coaptada e luxação da articulação femurotibial.

As miniplacas de titânio possuem características semelhantes às placas convencionais, todavia apresentam como vantagens o peso reduzido, a grande elasticidade e a maleabilidade. Essas características facilitam o retorcimento e a adaptação às superfícies ósseas (Bilgili e Kurum, 2003). Além disso, seu tamanho diminuto possibilita a utilização em aves de pequeno e médio porte, e o tamanho de seus parafusos causa menor lesão à cortical óssea (Hatt et al., 2001). As miniplacas, comparadas aos demais dispositivos, apresentam menor peso, facilitando a manutenção da deambulação do paciente, como foi observado neste caso.

Palavras-chave: ave, fratura, miniplaca, tibiotarso, silvestres

\begin{abstract}
Among the surgical problems of birds, fractures are the most prevalent, and the long bones of the wings and pelvic limbs are the most frequently affected. The success of orthopedic surgery in birds is directly related to the techniques used to undo the mechanical forces of a fracture. At the veterinary teaching hospital a psitacid from the Ara ararauna (blue-and-yellow-macaw) species with a clinical history of trauma in the cage was treated. Craniocaudal and lateral radiographs of the left limb demonstrated a mid-diaphyseal fracture of the tibiotarsus. The bird was sent to surgery. The patient was anesthetized with isufluorane and for the pre-anesthesic medicacion methadone was administrated. A medial surgical approach to the tibiotarsus was made and fracture reduction was performed. The fracture was stabilized through a titanium miniplate (system $2 \mathrm{~mm}$ ) fixed medially on the tibiotarsus with 6 cortical miniscrews, being 3 proximal and 3 distal to the fracture site. In this case, there was no complication in placing the miniplate and miniscrews, resulting in a satisfactory alignment of the fragments and causing an efficient reduction of the diaphyseal fracture of tibiotarsus with functional return of the limb and bone consolidation.
\end{abstract}

Keywords: bird, fracture, miniplate, tibiotarsus, wildlife 


\section{REFERÊNCIAS BIBLIOGRÁFICAS}

ALIEVI, M.M.; OLIVEIRA, A.N.C.; FERREIRA, P.A. et al. Osteossíntese de úmero em pombos domésticos (Columba livia) associando-se pinos metálicos e polimetilmetacrilato intramedulares após osteotomia diafisária. Arq. Bras. Med. Vet. Zootec., v.60, p.843-850, 2008.

ALIEVI, M.M.; SCHOSSLER, J.E.; HIPPLER, R.A. et al. Redução fechada e fixação esquelética externa tipo I para tratamento de fraturas de tibiotarso em pombos domésticos (Columa livia). Arq. Bras. Med. Vet. Zootec., v.54, p.259-266, 2002.

BILGILI, H.; KURUM, B. Treatment of fractures of the mandible and maxilla by mini titanium plate fixation systems in dogs and cats. Aust. Vet. J., v.81, p.671-673, 2003.

DAVIDSON, J.R.; MITCHELL, M.A.; RAMIREZ, S. Plate fixation of a coracoid fracture in a bald, eagle (Haliaeectus leucocephalus). J. Avian Med. Surg., v.19, p.303308, 2005.

FERRAZ, V.C.M.; FERRIGNO, C.R.A.; CORTOPASSI, S.R.G., et al. Avaliação radiográfica e de função de vôo após fixação de osteotomias distais do úmero em pombas (Columba livia), com modelo de fixador externo articulado. Pesqu. Vet. Bras., v.28, p.351-357, 2008.

GUZMAN, D.S.M.; BUBENIK, L.J.; LAUER, S.K., et al. Repair of a coracoid luxation and a tibiotarsal fracture in a bald eagle (Haliaeectus leucocephalus). J. Avian Med. Surg., v.21, p.188$195,2007$.
HATT, J.M.; SCHLÃPFER, I.N, WERTHERM, C.J. Verwerdung der maxillofazialen miniplatte compact 1.0 zur behandlung einer distalen tibiotarsusfraktur bei einem afrikanischen graupapaguei (Psittacus erithacus). Tierärztl Prax, v.29, p.135-138, 2001.

HELMER, P.; REDIG, P.T. Surgical resolutions of orthopedic disorders. In: HARRISON, G.J.; LIGHTFOOT, T.L. Clinical Avian Medicine. Palm Beach: Spix Publishing, 2006. v.2, p.761774.

HOLLAMBY, S.; DEJARDIN, L.M.; SIKARSKIE, J.G. et al. Tibiotarsal fracture repair in a bald eagle (Haliaeectus leucocephalus) using an interlocking nail. J. Zoo Wildl. Med., v.35, p.77-81, 2004.

STAUBER, E.; MULHOLLAND, J.A.; LEVINE, E.W. et al. Successful rehabilitation of a severely injuried peregrine falcon. J. Avian Med. Surg., v.22, p.346-350, 2008.

SUGAWARA, J.; BAIK, U.B.; UMEMORI, M. et al. Treatment and post treatment dentoalveolar changes following intrusion of mandibular molars with application of skeletal anchorage system (SAS) for open bite correction. Int. J. Adult Orthodon. Orthognath. Surg., v.17, p.243253, 2002.

WEST, P.G.; ROWLAND, G.R.; BUDSBERG, S.D. et al. Histomorphometric and angiographic analisys of bone healing in the humerus of pigeons. Am. J. Vet. Res., v.57, p.1010-1015, 1996. 